\title{
Validity and reliability of the Persian version of the Brief Aging Perceptions Questionnaire in Iranian older adults
}

\author{
Leila Sadegh Moghadam ${ }^{1,2}$ \\ Mahshid Foroughan ${ }^{1,3}$ \\ Farahnaz Mohammadi \\ Shahboulaghi ${ }^{1,4}$ \\ Fazlollah Ahmadi ${ }^{5}$ \\ Moosa Sajjadi ${ }^{6}$ \\ Akram Farhadi ${ }^{3}$
}

'Iranian Research Center on Aging, University of Social Welfare and

Rehabilitation Sciences, Tehran, Iran; ${ }^{2}$ Nursing Department, Gonabad

University of Medical Sciences, Gonabad, Iran; ${ }^{3}$ Department of Ageing,

University of Social Welfare and Rehabilitation, Tehran, Iran; ${ }^{4}$ Nursing Department, University of Social Welfare and Rehabilitation Sciences, Tehran, Iran; ${ }^{5}$ Nursing Department, Faculty of Medical Sciences, Tarbiat Modares University, Tehran, Iran; ${ }^{6}$ Department of Medical-Surgical Nursing, Faculty of Nursing and Midwifery, Gonabad University of Medical Sciences, Gonabad, Iran

Correspondence: Farahnaz Mohammadi Shahboulaghi

Iranian Research Center on Aging, University of Social Welfare and Rehabilitation Sciences, Kodakyar Avenue, Daneshjo Boulevard, Evin, I $9857-$ I 3834 Tehran, Iran

Tel +9821 22180008

Fax +98 21 2218 0121

Email f.mohammadi@uswr.ac.ir
This article was published in the following Dove Press journal:

Clinical Interventions in Aging

2 May 2016

Number of times this article has been viewed

Background: Perceptions of aging refer to individuals' understanding of aging within their sociocultural context. Proper measurement of this concept in various societies requires accurate tools.

Objective: The present study was conducted with the aim to translate and validate the Brief Aging Perceptions Questionnaire (B-APQ) and assess its psychometric features in Iranian older adults.

Method: In this study, the Persian version of B-APQ was validated for 400 older adults. This questionnaire was translated into Persian according to the Wild et al's model. The Persian version was validated using content, face, and construct (using confirmatory factor analysis) validities, and then its internal consistency and test-retest reliability were measured. Data were analyzed using the statistical software programs SPSS 18 and EQS-6.1.

Results: The confirmatory factor analysis confirmed construct validity and five subscales of B-APQ. Test-retest reliability with 3-week interval produced $r=0.94$. Cronbach's alpha was found to be 0.75 for the whole questionnaire, and from 0.53 to 0.77 for the five factors.

Conclusion: The Persian version of B-APQ showed favorable validity and reliability, and thus it can be used for measuring different dimensions of perceptions of aging in Iranian older adults.

Keywords: Brief Aging Perception Questionnaire, psychometric study, older adults, validity

\section{Introduction}

In today's modern world, socioeconomic development has led to reduced population growth and increased life expectancy, so that the growth of the older adult population exceeds that of the total population of the world.

Old age is one of the stages in human life, but given interpersonal relationships and the ruling cultural context, people have widely different biological, psychological, and social perceptions of old age. ${ }^{2}$ To learn about their health, well-being, personal identity, and consequences of individuals' behavior in their middle and old age, understanding their experiences and perceptions of old age is essential. . $^{3,4}$

Perception of aging refers to the elderly's understanding of their own physical and psychological conditions in old age, and it is a strong predictor of their health and mortality. ${ }^{5,6}$

Several studies have shown that people with a positive perception of aging have better health, longer life, fewer cardiovascular problems, and lower mortality compared to those without. ${ }^{7,8}$ Positive perception of aging leads to better quality of life, greater satisfaction with life, and less depression and loneliness. ${ }^{9,10}$ 
Barker et al were among the first people who introduced the Aging Perceptions Questionnaire (APQ) using the selfregulation model. ${ }^{9}$ Barker et al's questionnaire contains 32 items and seven subscales, and measures various dimensions of perceptions of aging. ${ }^{9}$ Aiming to reduce the burden of responding to many questions, given the low tolerance of the elderly to respond to lengthy questionnaires and the possible inappropriate or unreliable responses, Sexton et al introduced a shortened version of the questionnaire with 17 items and five dimensions with suitable psychometrics. ${ }^{6}$ Its dimensions include progressive course (awareness of the aging process over time), positive outcomes (positive outcomes of aging like getting wiser, more experienced, etc), negative outcomes and negative control (inappropriate outcomes of aging like lack of control over conditions), and emotional reactions (emotional responses to aging like depression, anger, etc). ${ }^{6}$

Owing to the importance of measuring perceptions of aging in the elderly, the questionnaire was soon translated to French, Dutch, Chinese, and other languages, and used in numerous studies. ${ }^{11-13}$

It is estimated that the elderly constitute $60 \%$ of health care system customers in Iran, as an aging country in coming years. ${ }^{14}$ Perception of aging among the elderly is a criterion for judging their health and life quality, but there is no valid and reliable questionnaire for this purpose in Iran. The lack of an effective questionnaire is a big gap in the studies on aging, and an important barrier to the pursuit of the elderly's status and effects of interventions.

Appropriate perceptions of aging and understanding of favorable or unfavorable behaviors of older adults facing various signs and complications associated with old age enables taking effective actions to prevent potential old age problems and reduce complications, and ultimately improve their quality of life. ${ }^{15-17}$

This study was conducted with the aim of translating the Brief Aging Perceptions Questionnaire (B-APQ) into Persian, and assess psychometric features of the Persian version.

\section{Methods}

The study protocol was approved by the Ethics Committee of the University of Social Welfare and Rehabilitation Sciences in Tehran, Iran (USWR.REC.1393.136). In the present methodological study, ${ }^{18}$ the B-APQ scale was translated into Persian and then the Persian version was validated in Iranian older adults. Participants were selected from older adults experiencing old age, living with their family and in the community in the city of Gonabad (Khorasan-Razavi Province, Iran). Gonabad, with $11.5 \%$ older adult population, is one of the oldest cities in Iran, with a special feature of urban and rural context next to each other. ${ }^{19}$

Sampling was performed from December 2014 to March 2015, and a sample size of 380 was determined based on Cochran's formula, which was increased to 420, taking into account possible $10 \%$ withdrawal, but ultimately 400 questionnaires were analyzed. A two-stage random cluster sampling method was used. Given that health centers in Gonabad cover $100 \%$ of the urban population, the cluster consisted of all four health centers in this town. Then, the number of older adults in each cluster was determined and a list of their names was prepared. Next, older adults from each center (identified according to the sample size required from each cluster using a table of random numbers) were identified and questioned.

Study inclusion criteria were: 60 years of age and older (start of old age in developing countries), willingness to take part in the study, and no history of treatment for psychiatric disorders (cognitive health with a score $\geq 7$ based on Abbreviated Mental Test [AMT]). ${ }^{20}$

In this study, translation and validation of the tool was performed according to Wild et al's method. ${ }^{21}$ Permission was obtained from the designer, and the original version was translated into Persian by two people fluent in English. The two translated versions were compared and after slight modifications in wording, the final version was obtained. Next, the final translated version was translated back into English by two people fluent in both Persian and English, who had no contact with the first translators. The backward translated version was emailed to the original designer, and was approved by him or her. Then, the questionnaire was validated using face, content, and construct (confirmatory factor analysis) validities, and its internal consistency and reliability was confirmed. For the purpose of content validity, modification comments about the questionnaire were sought from eight experts (one clinical psychologist, one psychiatrist, four assistant professors in nursing, and two assistant professors experienced in questionnaire design). The content validity index (CVI) was used for the quantitative part of the assessment and the scale's compliance with Persian grammar, its use of appropriate terminology, good phrasing, the scoring method used, and the responses given were then examined for the qualitative part of the assessment. Based on the index proposed by Waltz and Bausell, CVI $<0.7$ was unacceptable, $\mathrm{CVI}<0.78$ required modification and revision, and $\mathrm{CVI} \geq 0.79$ was acceptable. ${ }^{22,23}$

Afterward, the questionnaire was given to ten older adults to express their views on ease of use and understandability of sentences. The Persian version was confirmed and finalized with no particular modification of items. 
Following selection of eligible older adults, study objectives and method were explained to them and their written informed consents were obtained. Then, a demographic questionnaire (containing data about age, sex, education, marital status, occupation, and place of residence) and B-APQ were given to them to complete. For illiterate participants, the questionnaire was read and their answers were ticked by the researcher.

\section{Analysis of data}

Demographic data were assessed by SPSS 18 (SPSS Inc., Chicago, IL, USA) using descriptive statistics.

Assessment of construct validity of the Persian B-APQ was performed using confirmatory factor analysis in EQS-6.1 software (Multivariate software, Inc., Encino, CA, USA). Confirmatory factor analysis is a method of presenting structural equations used in determining goodness-of-fit between a theoretical model and data obtained from study samples. ${ }^{24}$ Compatibility of the model was determined using maximum probability algorithm. There are several goodness-of-fit indices for deciding compatibility of the model, and it is preferable to use several indices. ${ }^{25,26}$ In this study, goodness-of-fit indices used included: chi-square, root mean square error of approximation, goodness-of-fit index (GFI), comparative fit index, and adjusted GFI. Root mean square error of approximation is an important index, with values less than 0.08 indicating acceptability and values less than 0.05 indicating a good fit. ${ }^{24,26}$ Appropriate values for other indices include comparative fit index, GFI $>0.9$, and adjusted GFI $>0.85 .{ }^{27,28}$ Internal consistency and reliability was assessed with SPSS 18 software. Internal consistency of the scale was determined with Cronbach's alpha for the whole scale and each of the subscales, and reliability with Pearson's correlation coefficient between test-retest with 3 -week interval in 20 older adults. An interval of $2-3$ weeks between two tests is usually appropriate. ${ }^{29}$

\section{Results}

A total of 400 questionnaires were statistically analyzed. Table 1 presents the demographic details of participants.

Based on the views expressed by the eight experts, CVI was found to be $0.97,0.95$, and 0.96 for relevance, clarity, and simplicity of items of the scale, respectively. CVI for the five subscales: timeline chronic, consequences positive, control positive, consequences and control negative, and emotional representations was found to be 1.00, 0.87, 0.95, $0.97,0.95$, respectively.

\section{Reliability}

The reliability of the scale was assessed using the testretest and the internal consistency methods. The test-retest
Table I Demographic and clinical data of participating older adults

\begin{tabular}{lll}
\hline Categories & N & $\%$ \\
\hline Sex & 198 & \\
Female & 202 & 49.5 \\
$\quad$ Male & & 50.5 \\
Age & 291 & \\
60-75 (years) & 98 & 72.8 \\
$76-90$ (years) & 11 & 24.4 \\
90 (plus years) & & 2.8 \\
Marital status & 310 & \\
Married & 90 & 77.5 \\
Widow/widower & & 22.5 \\
Educational level & 155 & \\
Illiterate & 166 & 38.5 \\
Primary and guidance school & 23 & 41.3 \\
Under diploma & 24 & 5.8 \\
Diploma & 32 & 6.0 \\
University degree & & 8.1 \\
Living arrangements & 70 & \\
Alone & 188 & 17.5 \\
Spouse & 114 & 47.0 \\
Child and spouse & 28 & 28.5 \\
Child & & 7.0 \\
Disease history & 110 & 27.5 \\
Heart disease & 40 & 10.0 \\
Diabetes & 23 & 5.8 \\
Kidney disease & 167 & 25.0 \\
High blood pressure & 100 & \\
Other disease & & \\
\hline
\end{tabular}

correlation coefficient for B-APQ was found to be 0.948 $(P<0.001)$. Correlation coefficient for the subscales: timeline chronic, Positive consequences, Positive control, Negative consequences and control, and emotional representations was found to be $0.987,0.9,0.984,0.987$, and 0.95 respectively. Cronbach's alpha was calculated to assess the internal consistency of the scale. Cronbach's alpha was calculated as 0.75 for the whole scale and as $0.69,0.53,0.77,0.67$, and 0.70 for the subscales of timeline chronic, positive consequences, positive control, negative consequences and control, and emotional representations (Table 2).

Confirmatory factor analysis was performed for assessment of construct validity and providing the most appropriate model of B-APQ. Table 3 presents confirmatory factor analysis results.

Table 2 Internal consistency and test-retest reliability of the subscales

\begin{tabular}{lll}
\hline Subscale & $\begin{array}{l}\text { Cronbach's } \\
\text { alpha }\end{array}$ & $\begin{array}{l}\text { Test-retest } \\
\text { reliability }\end{array}$ \\
\hline Timeline chronic & 0.692 & 0.987 \\
Positive consequences & 0.538 & 0.900 \\
Positive control & 0.773 & 0.984 \\
Negative consequences and control & 0.673 & 0.987 \\
Emotional representations & 0.704 & 0.950 \\
Total & 0.759 & 0.948 \\
\hline
\end{tabular}


Table 3 Goodness-of-fit indices of confirmatory factor analysis of $B-A P Q$

\begin{tabular}{llllll}
\hline$\chi^{2}(d f), P$-value & RMSEA $(90 \% \mathrm{Cl})$ & CFI & GFI & AGFI & RMR \\
\hline
\end{tabular}

\begin{tabular}{llllll}
\hline $261.34(d f),<0.001$ & $0.06(0.5 \mathrm{I}-0.69)$ & 0.91 & 0.93 & 0.90 & 0.75
\end{tabular}

Abbreviations: B-APQ, Brief Aging Perceptions Questionnaire; $d f$, degrees of freedom; RMSEA, root mean square error of approximation; $\mathrm{Cl}$, confidence interval; $\mathrm{CFI}$, comparative fit index; GFI, goodness-of-fit index; AFGl, adjusted goodness-of-fit index; RMR, root mean square residual.

\section{Discussion and conclusion}

Perception of aging is a concept that can have several effects on older adults, and its study is hugely important. But, despite its importance, it has not been studied in Iran, perhaps due to lack of an appropriate and credible tool. This study was conducted with the aim to translate and validate the B-APQ in older adults. In this study, B-APQ was translated into Persian, and its psychometric features and factor structure were assessed. Validation of a tool is necessary when it is going to be used in a different society and culture, since it may not be appropriate for the particular society. ${ }^{30,31}$ In this study, content validity, construct validity, and reliability of this tool were assessed, and results obtained showed appropriate validity and reliability for the Persian version of B-APQ, with similar structure of its dimensions to the original version. ${ }^{6}$

This study confirmed a favorable content validity $(0.95)$ for the Persian version of B-APQ. CVI $\geq 0.9$ indicates appropriate value of the standard content validity of scales. ${ }^{29}$ Confirmatory factors analysis of B-APQ was performed using a sample of 400 older adults. The goodness-of-fit indices of the model indicated appropriateness of the 17 items and five dimensions of the Persian version model of B-APQ. But, achieving better compatibility and higher values of goodness-of-fit indices requires changes in the original scale. In confirmatory factor analysis through EQS, suggestions are made to modify and increase compatibility of the model, which may be implemented if logically and theoretically justified. ${ }^{24}$ In the Persian version, item four: "I get wiser as I get older" could have been eliminated due to a lower correlation coefficient than other items. But, since only two items would have remained in the dimension "positive consequences", it was decided to keep this item. In other studies that performed psychometric assessment of B-APQ, none of the items were eliminated. ${ }^{12}$ In this study, reliability coefficient of "positive consequences" was lower than other dimensions $(\alpha=0.53)$. Similarly, in a study by Haghi Ascarabadi, aiming to validate the APQ developed by Barker et al, the "positive consequences" dimension showed the lowest correlation. ${ }^{32}$ This is probably due to the fact that for Iranian older adults, positive consequences of aging are not as tangible as other dimensions, and negative stereotypes of aging reduce consideration of positive aspects, or else, they may find positive consequences in other issues not dealt with in this study. In studies by Sexton et al and Chen et al, the dimension of positive consequences had a lower value of Cronbach's alpha (still within the acceptable range). ${ }^{6,13}$

The Cronbach's alpha of 0.75 for the whole questionnaire confirmed appropriate internal consistency of the Persian version of B-APQ. Cronbach's alpha is the best standard used for assessment of internal consistency reliability. Cronbach's alpha of $\sim 0.7$ appears sufficient, and values of 0.8 and greater indicate high internal consistency of the tool. ${ }^{29}$ In the present study, the values of Cronbach's alpha obtained for the whole scale and for its dimensions were comparable to those found in a study by Slotman et al in older adults in the Netherlands. ${ }^{12}$

As well as internal consistency, test-retest results also showed favorable reliability for the Persian version of this questionnaire $(P=0.001, r=0.948)$.

In this study, strong points included large sample size and random sampling, and limitations included illiteracy of $-38.8 \%$ of participants and completion of questionnaires by a researcher through interviews, and also, sampling in a limited area, which may not represent the whole Iranian older adult community. A limitation in the present study was the lack of a standard Persian scale for concurrent validity. In the Persian version, although some of the items with low correlation coefficients were not eliminated (due to small number of items in those dimensions), it is recommended that with future qualitative studies and better understanding of older adults, items eliminated be replaced by other culturally compatible items. The present study's results confirmed appropriate validity and reliability of the Persian version of B-APQ as well as its five dimension structure. It can therefore be used in assessment of perception of aging in Iranian older adults. Use of this questionnaire by gerontologists, nurses, caregivers, and researchers can improve their knowledge of aging and enhance quality of life of the older adults, increase satisfaction, and improve care of older adults. These studies will constitute cornerstones of future studies on perception of aging in Iran. Given the cognitive nature of perception of aging, further qualitative studies in this area are required to identify its other possible dimensions.

\section{Acknowledgments}

The present study was part of a $\mathrm{PhD}$ thesis in gerontology approved by the Gerontology Research Center of the Welfare and Rehabilitation Sciences University (Tehran-Iran) in partnership with Gonabad University of Medical Sciences 
(Khorasan-Razavi-Iran). We hereby wish to express our thanks to the authorities of both universities and also authorities of health centers in Gonabad city. We also wish to especially thank all participating older adults.

\section{Disclosure}

The authors report no conflicts of interest in this work.

\section{References}

1. Mirfallah Nassiri N. Demographic determinants of population ageing and related indicators in Iran. IJOSS. 2008;18(2):1-14.

2. de Freitas MC, Queiroz TA, de Sousa JA. The meaning of old age and the aging experience of in the elderly. Rev Esc Enferm USP. 2010;44(2): 407-412.

3. Demakakos P, Hacker E, Gjonça E. Perceptions of ageing. In: Banks J, Breeze E, Lessof C, Nazroo J, editors. Retirement, Health and Relationships of the Older Population in England: The 2004 English Longitudinal Study of Ageing (Wave 2). London: The Institute for Fiscal Studies; 2006:339-351.

4. Rice PF. Human Development: A Life-Span Approach. New York: Macmillan Publishing Company; 1992.

5. International Longevity Center. [webpage on the Internet]. Available from: http://www.ilc-alliance.org/. Accessed February 22, 2016.

6. Sexton E, King-Kallimanis BL, Morgan K, McGee H. Development of the brief ageing perceptions questionnaire (B-APQ): a confirmatory factor analysis approach to item reduction. BMC Geriatr. 2014;14:44.

7. Levy BR, Slade MD, Kunkel SR, Kasl SV. Longevity increased by positive self-perceptions of aging. J Pers Soc Psychol. 2002;83(2):261-270.

8. Kotter-Grühn D, Kleinspehn-Ammerlahn A, Gerstorf D, Smith J. Self-perceptions of aging predict mortality and change with approaching death: 16-year longitudinal results from the Berlin Aging Study. Psychol Aging. 2009;24(3):654-667.

9. Barker M, O’Hanlon A, McGee HM, Hickey A, Conroy RM. Crosssectional validation of the Aging Perceptions Questionnaire: a multidimensional instrument for assessing self-perceptions of aging. $B M C$ Geriatr. 2007;7:9.

10. Steverink N, Westerhof GJ, Bode C, Dittmann-Kohli F. The personal experience of aging, individual resources, and subjective well-being. J Gerontol B Psychol Sci Soc Sci. 2001;56(6):P364-P373.

11. Ingrand I, Houeto JL, Gil R, Mc Gee H, Ingrand P, Paccalin M. The validation of a French-language version of the Aging Perceptions Questionnaire (APQ) and its extension to a population aged 55 and over. BMC Geriatr. 2012;12:17.

12. Slotman A, Cramm JM, Nieboer AP. Validation of the Dutch Aging Perceptions Questionnaire and development of a short version. Health Qual Life Outcomes. 2015;13:54.

13. Chen X, Hu Y, Zhu D, Li J, Zhou L. Chinese version of the Aging Perceptions Questionnaire (C-APQ): assessment of reliability and validity. Aging Ment Health. Epub 2015 Apr 8.

14. Karami Matin B, Rezaei S, SHaahmadi F, Kazemi Karyani A. [Ageing in Iran in 1410, a warning to health care system]. Teb va Tazkieh. 2012; 22(2):9-18. Persian. Available from: http://tazkiyeh.behdasht.gov.ir/ uploads/Salmandi_Summer92.pdf. Accessed March 23, 2016.
15. Löckenhoff CE, De Fruyt F, Terracciano A, et al. Perceptions of aging across 26 cultures and their culture-level associates. Psychol Aging. 2009;24(4):941-954.

16. Musaiger AO, D'Souza R. Role of age and gender in the perception of aging: a community-based survey in Kuwait. Arch Gerontol Geriatr. 2009;48(1):50-57.

17. Yun RJ, Lachman ME. Perceptions of aging in two cultures: Korean and American views on old age. J Cross Cult Gerontol. 2006;21(1-2): $55-70$.

18. Rose-Grippa K, Gorney-Moreno MJ. Study guide: Nursing research: Methods and critical appraisal for evidence-based practice. 6th ed. St Louis: Mosby; 2006.

19. Gonabad ranked first in the country in aging [webpage on the Internet]. Nobat.ir; 2016. Available from: http://tasalamati.com/e/PRnZ/. Accessed March 23, 2016

20. Bakhtiari F, Foroughan M, Fakhrzadeh H, et al. Validation of the Persian version of Abbreviated Mental Test (AMT) in elderly residents of Kahrizak Charity Foundation. Iranian Journal of Diabetes and Lipid Disorders. 2014;13(6):487-494.

21. Wild D, Grove A, Martin M, et al. Principles of good practice for the translation and cultural adaptation process for patient-reported outcomes (PRO) measures: report of the ISPOR Task Force for Translation and Cultural Adaptation. Value Health. 2005;8(2):94-104.

22. Polit DF, Beck CT. The content validity index: are you sure you know what's being reported? Critique and recommendations. Res Nurs Health. 2006;29(5):489-497.

23. Polit DF, Beck CT, Owen SV. Is the CVI an acceptable indicator of content validity? Appraisal and recommendations. Res Nurs Health. 2007; 30(4):459-467.

24. Kline RB. Principles and Practice of Structural Equation Modeling New York, NY: Guilford Publications Inc; 2005.

25. Brown TA. Confirmatory Factor Analysis for Applied Research. 1st ed. The Guilford Press; 2006.

26. Seo DC, Torabi MR, Blair EH, Ellis NT. A cross-validation of safety climate scale using confirmatory factor analytic approach. J Safety Res. 2004;35(4):427-445.

27. Bentler PM. Comparative fit indexes in structural models. Psychol Bull. 1990;107(2):238-246

28. Helsen K, Van den Bussche E, Vlaeyen JW, Goubert L. Confirmatory factor analysis of the Dutch Intolerance of Uncertainty Scale: Comparison of the full and short version. J Behav Ther Exp Psychiatry. 2013; 44(1):21-29.

29. Polit DF, Beck CT. Essentials of Nursing Research: Appraising Evidence for Nursing Practice. Lippincott Williams \& Wilkins; 2013.

30. Michaeli Manee F. Internal consistency and confirmatory factor analysis of Wells and Davis Thought control questionnaire. Iranian Journal of Psychiatry and Clinical Psychology. 2011;16(4):468-478.

31. Hillen MA, Butow PN, Tattersall MH, et al. Validation of the English version of the Trust in Oncologist Scale (TiOS). Patient Educ Couns. 2013;91(1):25-28

32. Haghi Ascarabadi F. Translation and validation of aging perception questionnaire [master's thesis]. Karaj: Karaj Azad University; 2012.
Clinical Interventions in Aging

\section{Publish your work in this journal}

Clinical Interventions in Aging is an international, peer-reviewed journal focusing on evidence-based reports on the value or lack thereof of treatments intended to prevent or delay the onset of maladaptive correlates of aging in human beings. This journal is indexed on PubMed Central, MedLine,

\section{Dovepress}

CAS, Scopus and the Elsevier Bibliographic databases. The manuscript management system is completely online and includes a very quick and fair peer-review system, which is all easy to use. Visit http://www.dovepress. com/testimonials.php to read real quotes from published authors. 